\title{
Modelling of the Atmospheric Corrosion of Copper in the Province of Las Palmas. Studies Using Classic and Electrochemical Techniques
}

\author{
P.M. González, ${ }^{1, *}$ D.M. Mos, ${ }^{2}$ F.J. Santana, ${ }^{2}$ J. Vaswani, ${ }^{2}$ \\ J.J. Santana, ${ }^{2}$ J.E. González ${ }^{2, *}$ \\ 1) Dpto. Cartografía y Expresión Gráfica en la Ingeniería, Universidad de Las Palmas de Gran \\ Canaria, Campus universitario de Tafira. C.P. 35017. Las Palmas de Gran Canaria-Islas \\ Canarias - España \\ 2) Dpto. Ingeniería de Procesos, Grupo CAFMA. Campus universitario de Tafira. C.P. 35017, \\ Las Palmas de Gran Canaria - Islas Canarias - España
}

Received $18^{\text {th }}$ June 2007; accepted $20^{\text {th }}$ January 2008

\begin{abstract}
Simulation of the effects of natural atmospheres on metals using electrochemical techniques together with accelerated tests presents certain characteristics that make it highly interesting from the point of view of the study and prediction of corrosion. It will enable us to forecast the behavior of a metal exposed to a particular environment without the need to perform field test, with a consequent saving in terms of time and financial expenses. The aim of this study is to establish the correlation between the atmospheric corrosion rate of copper (obtained by atmospheric exposure at stations located in the province of Las Palmas, Spain) with the different variables that intervene in the corrosion process. In the same way, for the determination of the mathematical model that best fits the results obtained experimentally and which takes into account the most influential parameters in the corrosion process, the power Law model has been used which generically reproduces the electrochemical process which occurs in atmospheric corrosion.
\end{abstract}

Keywords: atmospheric corrosion, copper, polarization, mathematical modelling.

\section{Introducción}

Dentro de los diferentes tipos de corrosión una de las más importantes es la corrosión atmosférica. Las Islas Canarias destacan sobre cualquier otro emplazamiento geográfico por confluir en una pequeña extensión de terreno hasta 8 microclimas distintos, por lo que nos encontramos con todos los

\footnotetext{
*Corresponding author. E-mail address: pgonzalez@dcegi.ulpgc.es; jgonzalez@dip.ulpgc.es
} 
ambientes corrosivos posibles, desde los específicos para zonas tropicales, pasando por ambientes marino - industriales hasta ambiente rural. Los factores de mayor influencia en el aumento de la velocidad de corrosión son: el primero y más importante el aerosol marino, que contiene iones cloruros y sulfatos, la actividad industrial que genera sulfatos, el régimen de las lluvias así como la humedad del ambiente que forma finas películas sobre las superficies de los metales. La compleja relación entre estos factores durante el proceso de corrosión hace que los ensayos más utilizados a la hora de determinar el grado de corrosión de un metal en una determinada zona, sea la exposición de muestras del metal estudiado en estaciones de corrosión. En este sentido se han utilizado los datos aportados por el desarrollo del Mapa de Corrosividad de Canarias como datos de partida para el presente trabajo [3, 4].

En atmósferas limpias el cobre presenta velocidades de corrosión despreciables, aunque a medida que introducimos parámetros de influencia, la velocidad de corrosión aumenta considerablemente, tal y como se podrá observar con posterioridad. El ión cloruro es el parámetro que ejerce una mayor influencia en la velocidad de corrosión del cobre $[9,11]$. A pesar de ello, es difícil diferenciar en qué porcentaje influyen la presencia de cloruros y el aporte de humedad por el aerosol marino.

Los modelos para predecir el deterioro por corrosión de los metales en la atmósfera son de gran utilidad para conocer el efecto de las variables ambientales en la cinética del proceso corrosivo y se basan, generalmente, en la aplicación de la función potencial [8].

Intentar reproducir las relaciones entre los factores que afectan al proceso corrosivo en laboratorio es un trabajo complejo. Mediante técnicas electroquímicas se ha podido determinar la influencia relativa de los contaminantes más frecuentes en nuestra atmósfera, como los iones cloruros y sulfato y luego establecer una relación entre estos resultados y los obtenidos por el método gravimétrico. No se ha optado por caracterizar la corrosión atmosférica por vía electroquímica, sino determinar el efecto relativo de los agentes agresivos sobre las probetas de cobre y relacionar estos resultados cualitativamente con los obtenidos por el método gravimétrico.

El método más reciente de evaluar la velocidad de corrosión es el método de la resistencia de polarización, conocido también con el nombre de sus autores, Stern y Geary, [5]. Otra característica de los métodos electroquímicos es que suministran en todo momento información sobre la velocidad de corrosión instantánea, expresada como densidad de corriente. Por añadidura, permiten reproducir en multitud de sistemas reales metal/medio las condiciones de servicio, sin perturbar la corrosividad del medio, al multiplicar la concentración del agente agresivo por varios órdenes de magnitud.

De la misma manera los estudios electroquímicos nos permitirán analizar la posible presencia de procesos corrosivos localizados, sometiendo al metal a barridos de potencial crecientes. 


\section{Trabajo experimental}

\section{Corrosión atmosférica}

En las tres islas que conforman la provincia de Las Palmas, objeto del estudio, se han distribuido 39 estaciones de corrosión, de ellas 6 en Lanzarote, otras 6 en la isla de Fuerteventura y en Gran Canaria 27 estaciones, tal y como se muestra en la Fig. 1. En la tabla 1 se muestra las características de las probetas de cobre utilizadas en el estudio.
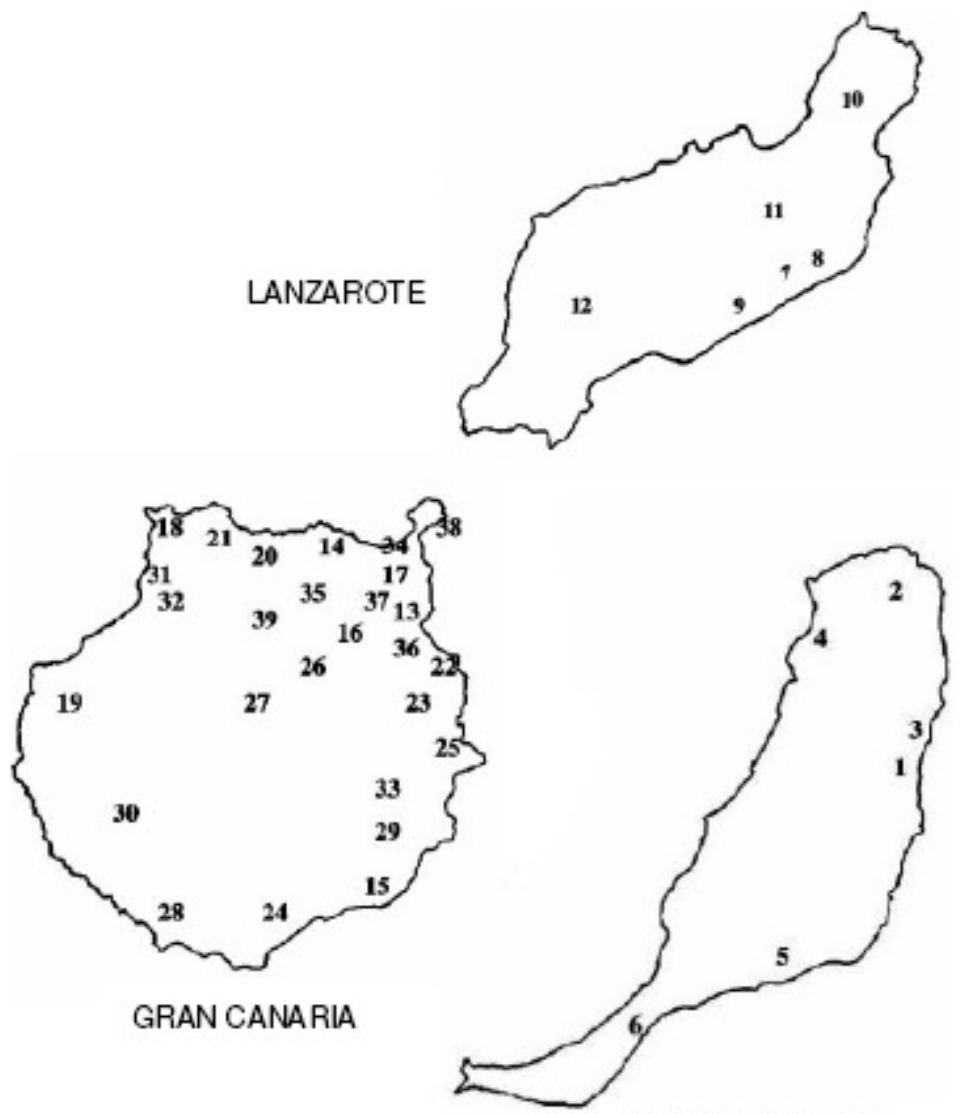

FUERTEVENTURA

Figura 1. Distribución de las estaciones de corrosión en la provincia de Las Palmas.

Tabla 1. Composición de las probetas de cobre.

\begin{tabular}{|c|c|c|c|c|c|c|c|c|}
\hline \multicolumn{6}{|c|}{ Composición química de las probetas de cobre (\%) } \\
\hline $\mathrm{Si}$ & $\mathrm{Cu}$ & $\mathrm{Mn}$ & $\mathrm{Mg}$ & $\mathrm{Zn}$ & $\mathrm{Ti}$ & $\mathrm{Fe}$ & $\mathrm{Cr}$ & otros \\
\hline 0.25 & 98.5 & 0.05 & 0.05 & 0.09 & 0.05 & 0.9 & - & 0.09 \\
\hline
\end{tabular}

La evaluación de la velocidad de corrosión se efectuó mediante pérdida de peso de las probetas recolectadas durante el primer año de exposición, utilizando el método gravimétrico para posteriormente obtener los modelos matemáticos que relacionaran la velocidad de corrosión con los niveles de sulfatos, cloruros y el tiempo de humectación (TDH) determinados en cada estación.

La limpieza de las probetas objeto del estudio fue realizada según norma internacional ASTM G1-90 [6]. 
Según establece esta norma, para la limpieza química se ha utilizado una disolución de $\mathrm{HCl}$ en agua destilada 1:1. Se limpian todas las probetas con agua y jabón, raspando con un cepillo para eliminar restos de polvo, grasa, etc. Después de efectuar la limpieza química se vuelven a sumergir las probetas en agua, se raspan con un cepillo, se pasan por agua destilada caliente, se lavan con acetona para eliminar el agua existente y se colocan en estufa un mínimo de 2 horas a 60 ${ }^{\circ} \mathrm{C}$. Finalmente se colocan en los desecadores hasta que se enfríen y se pesan, repitiendo todo proceso de limpieza hasta observar un peso constante.

La determinación de las categorías de corrosividad correspondientes a cada estación se obtuvo de los valores de velocidad de corrosión para el cobre correspondientes al primer año de exposición, según establece la norma ISO $9223[7]$.

\section{Modelación matemática}

Para la determinación del modelo matemático que mejor se ajuste a los resultados obtenidos experimentalmente se analizaron los resultados con la ecuación de la ley potencial (ecuación 1), modelo que de forma genérica reproduce el proceso electroquímico que se desarrolla en la corrosión atmosférica. De cara a tener en cuenta las particularidades de la zona geográfica objeto del estudio se modelaron los datos obtenidos con otras ecuaciones que incluyeran la influencia de los contaminantes descritos y la humedad de cara a evaluar la incidencia relativa de cada uno de ellos.

El modelo que, en base al coeficiente de correlación, mejor se ajustó a los datos obtenidos en las estaciones es el descrito como ecuación 2 en su expresión logarítmica. En ella se ha pretendido valorar de una manera sencilla la correlación existente entre el logaritmo de la velocidad de corrosión y los niveles de agentes agresivos junto con el de TDH y el tiempo de exposición.

El tiempo de humectación TDH está definido como el número de horas al año en las que la humedad relativa es superior al $80 \%$ y la temperatura es mayor de $0{ }^{\circ} \mathrm{C}$ [11]. Hemos determinado el tiempo de humectación con los datos suministrados por el Instituto Nacional de Meteorología.

$$
\begin{gathered}
\log (P)=a+b \cdot \log (t) \\
\log (P)=a+b \cdot \log (t)+k_{1} \cdot\left[\mathrm{Cl}^{-}\right]+k_{2} \cdot\left[\mathrm{SO}_{2}\right]+k_{3} \cdot[\mathrm{TDH}]
\end{gathered}
$$

donde: $\mathrm{P}=$ velocidad de corrosión en $\mu \mathrm{m} / \mathrm{año}, \mathrm{a}, \mathrm{b}, \mathrm{k}_{1}, \mathrm{k}_{2}, \mathrm{k}_{3}=$ constantes, $\left[\mathrm{SO}_{2}\right]$ = concentración de dióxido de azufre en $\mathrm{mg} /\left(\mathrm{m}^{2} \cdot\right.$ día $),\left[\mathrm{Cl}^{-}\right]=$concentración de cloruros en $\mathrm{mg} /\left(\mathrm{m}^{2} \cdot\right.$ día $), \mathrm{TDH}=$ tiempo de humectación (horas/año), $\mathrm{t}=$ tiempo de exposición, años. 


\section{Estudios electroquímicos}

El comportamiento electroquímico del cobre se ha estudiado mediante las técnicas en corriente continua, determinando el potencial de corrosión y la resistencia de polarización del cobre en medios con concentraciones variables de cloruros y sulfatos por separado, en un rango que oscila entre $0.1 \mathrm{M}$ hasta $1 \mathrm{M}$, así como con mezcla de ambos, variando la concentración de cloruros frente a concentración de sulfato fija de $0.05,0.1$ y $0.2 \mathrm{M}$.

Antes de iniciar las medidas electroquímicas, cada placa de cobre fue sometida a un proceso de pulido con papel esmeril de grados 500, 800 y 1000 y posterior pulido con pulidora mecánica y polvo de alúmina hasta calidad espejo para evitar posibles distorsiones en la medida. Luego se limpiaron con agua destilada.

Las medidas electroquímicas se realizaron en una célula electroquímica convencional de vidrio Pirex preparada para el montaje de tres electrodos, cuyas funciones son:

- trabajo con un área circular de $5.56 \mathrm{~cm}^{2}$, referencia y contraelectrodo;

- el electrodo de referencia empleado es el de $\mathrm{Ag}-\mathrm{AgCl}$ y como

contraelectrodo se utilizó un electrodo de platino (Fig. 2).

Para las medidas electroquímicas se ha utilizado un potenciostato/galvanostato EG\&G Princeton Applied Research, modelo 263 A.

Los electrodos se introdujeron en las disoluciones de contaminantes bajo estudio permitiendo que durante 30 minutos éstos pudieran alcanzar su correspondiente potencial de circuito abierto o potencial de corrosión $\mathrm{E}_{\text {corr. }}$ Seguidamente se impuso al electrodo, bajo este valor de potencial de corrosión un barrido de potencial de $\pm 250 \mathrm{mV}$ que permitió calcular la intensidad de corrosión (Io) y la resistencia de polarización $(\mathrm{Rp})$.

Con el fin de determinar la posible aparición del pitting como consecuencia de la presencia del ión cloruro, las probetas se sometieron a barridos de potencial próximos a $500 \mathrm{mV}$ por encima del potencial de corrosión [14].

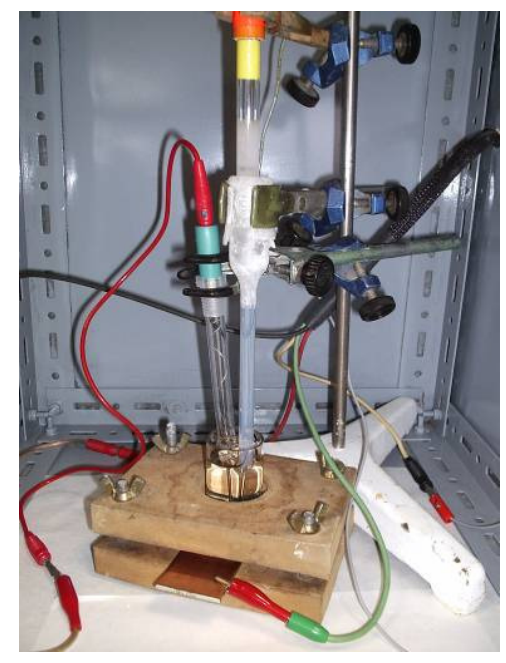

Figura 2. Conexión celda electroquímica. 


\section{Resultados obtenidos}

\section{Resultados corrosión atmosférica}

En las tablas 2 a 4 se muestran los valores de velocidad de corrosión para el cobre correspondientes al primer año de exposición, valores a partir de los cuáles se obtuvieron las categorías de corrosividad correspondientes a cada estación según normativa, tanto por pérdida de peso como por parámetros medioambientales.

Tabla 2. Categoría de las estaciones de la isla de Fuerteventura.

\begin{tabular}{|c|c|c|c|c|}
\hline \multirow[b]{2}{*}{ Estación } & \multicolumn{2}{|c|}{ Datos de velocidad del $1^{\mathrm{er}}$ año } & \multicolumn{2}{|c|}{ Categoría de corrosividad } \\
\hline & $\mathrm{g} /\left(\mathrm{m}^{2} \cdot \mathrm{año}\right)$ & $\mu / a n ̃ o$ & Pérdida de peso & Parámetros ambientales \\
\hline 1 & 51 & 6.15 & C5 & $\mathrm{C} 3$ o $\mathrm{C} 4$ \\
\hline 2 & 55 & 6.63 & $\mathrm{C} 5$ & C3 \\
\hline 3 & 84 & 10.25 & $\mathrm{C} 5$ & $\mathrm{C} 5$ \\
\hline 4 & 45 & 5.45 & $\mathrm{C} 5$ & $\mathrm{C} 3$ \\
\hline 5 & 54 & 6.55 & $\mathrm{C} 5$ & $\mathrm{C} 3$ \\
\hline 6 & 36 & 4.34 & $\mathrm{C} 5$ & $\mathrm{C} 3$ \\
\hline
\end{tabular}

Tabla 3. Categoría de las estaciones de la isla de Lanzarote.

\begin{tabular}{|c|c|c|c|c|}
\hline \multirow{2}{*}{ Estación } & \multicolumn{2}{|}{ Datos de velocidad del $1^{\mathrm{er}}$ año } & \multicolumn{2}{|c|}{ Categoría de corrosividad } \\
\cline { 2 - 5 } & $\mathrm{g} /\left(\mathrm{m}^{2} \cdot\right.$ año $)$ & $\mu /$ año & Pérdida de peso & Parámetros ambientales \\
\hline 7 & 32 & 3.93 & $\mathrm{C} 5$ & $\mathrm{C} 3$ \\
\hline 8 & 50 & 6.13 & $\mathrm{C} 5$ & $\mathrm{C} 3$ \\
\hline 9 & 52 & 6.38 & $\mathrm{C} 5$ & $\mathrm{C} 3$ \\
\hline 10 & 40 & 4.81 & $\mathrm{C} 5$ & $\mathrm{C} 3$ \\
\hline 11 & 50 & 6.07 & $\mathrm{C} 5$ & $\mathrm{C} 3$ \\
\hline 12 & 45 & 5.45 & $\mathrm{C} 5$ & $\mathrm{C} 3$ \\
\hline
\end{tabular}


Tabla 4. Categoría de las estaciones de la isla de Gran Canaria.

\begin{tabular}{|c|c|c|c|c|}
\hline \multirow{3}{*}{ Estación } & \multirow{2}{*}{\multicolumn{2}{|c|}{ Datos de velocidad del $1^{\mathrm{er}}$ año }} & \multicolumn{2}{|c|}{ Categoría de corrosividad } \\
\hline & & & \multirow{2}{*}{ Pérdida de peso } & \multirow{2}{*}{ Parámetros ambientales } \\
\hline & $\mathrm{g} /\left(\mathrm{m}^{2} \cdot \mathrm{año}\right)$ & $\mu / a$ ño & & \\
\hline 13 & 76 & 9.21 & C5 & C4 \\
\hline 14 & 15 & 1.82 & $\mathrm{C} 4$ & $\mathrm{C} 3$ \\
\hline 15 & 65 & 7.88 & $\mathrm{C} 5$ & $\mathrm{C} 3$ \\
\hline 16 & 5 & 0.60 & $\mathrm{C} 2$ & $\mathrm{C} 3$ \\
\hline 17 & 26 & 3.22 & $\mathrm{C} 5$ & C3 \\
\hline 18 & 55 & 6.63 & $\mathrm{C} 5$ & $\mathrm{C} 3$ \\
\hline 19 & 37 & 4.56 & $\mathrm{C} 5$ & $\mathrm{C} 3$ \\
\hline 20 & 21 & 2.53 & $\mathrm{C} 4$ & $\mathrm{C} 3$ \\
\hline 21 & 47 & 5.69 & $\mathrm{C5}$ & C3 \\
\hline 22 & 155 & 18.88 & $\mathrm{C} 5$ & $\mathrm{C} 5$ \\
\hline 23 & 33 & 3.98 & $\mathrm{C} 5$ & $\mathrm{C} 3$ \\
\hline 24 & 29 & 3.54 & $\mathrm{C} 5$ & $\mathrm{C} 3$ \\
\hline 25 & 63 & 7.69 & $\mathrm{C} 5$ & $\mathrm{C} 3$ \\
\hline 26 & 2 & 0.25 & $\mathrm{C} 2$ & C3 \\
\hline 27 & 7 & 0.85 & C3 & C3 \\
\hline 28 & 82 & 10.01 & $\mathrm{C} 5$ & $\mathrm{C} 3$ \\
\hline 29 & 36 & 4.34 & $\mathrm{C5}$ & C3 \\
\hline 30 & 24 & 2.94 & $\mathrm{C} 5$ & C3 \\
\hline 31 & 36 & 4.38 & $\mathrm{C} 5$ & C3 \\
\hline 32 & 18 & 2.15 & $\mathrm{C} 4$ & C3 \\
\hline 33 & 21 & 2.57 & $\mathrm{C} 4$ & C3 \\
\hline 34 & 39 & 4.79 & $\mathrm{C} 5$ & C3 \\
\hline 35 & 4 & 0.51 & $\mathrm{C} 2$ & C3 \\
\hline 36 & 24 & 2.91 & $\mathrm{C} 5$ & C3 \\
\hline 37 & 27 & 3.27 & $\mathrm{C5}$ & C3 \\
\hline 38 & 72 & 8.76 & $\mathrm{C5}$ & C3 \\
\hline 39 & 15 & 1.82 & $\mathrm{C} 4$ & $\mathrm{C} 4$ \\
\hline
\end{tabular}

\section{Resultados modelación matemática}

En las tablas 5 a la 8 se muestran los resultados obtenidos al realizar el modelado matemático de las estaciones de forma individual y agrupada por categorías utilizando la ley potencial o bilogarítmica. 
Tabla 5. Modelación con la ley bilogarítmica.

\begin{tabular}{|c|c|c|c|}
\hline \multirow{2}{*}{ Estación } & \multicolumn{2}{|c|}{$\log (\mathrm{P})=\mathrm{a}+\mathrm{b} \cdot \log (\mathrm{t})$} & \multirow{2}{*}{$\mathrm{r}^{2}$} \\
\cline { 2 - 3 } & $\mathrm{a}$ & $\mathrm{b}$ & \\
\hline 1 & 0.7442 & 0.5136 & 0.9716 \\
\hline 2 & 0.8018 & 0.7567 & 0.9336 \\
\hline 3 & 0.9845 & 0.6057 & 0.9244 \\
\hline 4 & 0.7438 & 0.6190 & 0.7027 \\
\hline 5 & 0.7746 & 0.8657 & 0.9807 \\
\hline 6 & 0.7127 & 0.5003 & 0.9047 \\
\hline 7 & 0.5099 & 0.7827 & 0.8560 \\
\hline 8 & 0.7755 & 0.8829 & 0.9695 \\
\hline 9 & 0.8004 & 0.4944 & 0.9716 \\
\hline 10 & 0.6781 & 0.7111 & 0.9258 \\
\hline 11 & 0.7273 & 0.7426 & 0.9235 \\
\hline 12 & 0.7012 & 0.7954 & 0.8995 \\
\hline 13 & 0.9241 & 0.6933 & 0.9397 \\
\hline 14 & 0.0701 & 0.8570 & 0.7315 \\
\hline 15 & 0.8605 & 0.2941 & 0.9276 \\
\hline 16 & 0.3393 & 0.8738 & 0.7606 \\
\hline 17 & 0.3784 & 0.9773 & 0.9011 \\
\hline 18 & 0.7117 & 0.8364 & 0.9369 \\
\hline 19 & 0.4860 & 0.7670 & 0.5772 \\
\hline 20 & 0.1955 & 0.5723 & 0.8023 \\
\hline 21 & 0.6440 & 0.8264 & 0.9244 \\
\hline 22 & 1.2419 & 0.6058 & 0.9958 \\
\hline 23 & 0.4981 & 0.7743 & 0.9594 \\
\hline 24 & 0.4410 & 0.7155 & 0.9356 \\
\hline 25 & 0.7792 & 0.4375 & 0.8793 \\
\hline 26 & 1.0025 & 1.2990 & 0.8228 \\
\hline 27 & 0.0213 & 0.1978 & 0.3709 \\
\hline 28 & 0.9488 & 0.8822 & 0.9896 \\
\hline 29 & 0.5395 & 0.4129 & 0.6275 \\
\hline 30 & 0.4010 & 0.3047 & 0.7797 \\
\hline 31 & 0.5524 & 0.7504 & 0.9103 \\
\hline 32 & 0.2163 & 0.3519 & 0.8123 \\
\hline 33 & 0.2875 & 0.8212 & 0.9388 \\
\hline 34 & 0.5265 & 0.9912 & 0.9443 \\
\hline 35 & 0.0259 & 0.4479 & 0.6211 \\
\hline 36 & 0.2634 & 0.6598 & 0.9111 \\
\hline 37 & 0.4067 & 0.4735 & 0.6166 \\
\hline 38 & 0.8078 & 0.5173 & 0.7938 \\
\hline 39 & 0.0223 & 0.3033 & 0.7007 \\
\hline & & & \\
\hline
\end{tabular}


Tabla 6. Modelación por categorías según pérdida de peso.

\begin{tabular}{|c|c|c|c|c|}
\hline \multicolumn{5}{|c|}{$\log (P)=a+b \cdot \log (t)$} \\
\hline Categoría (perdida peso) & Variabl & Coefficient & Standard error & t-ratio \\
\hline \multirow{2}{*}{$C 1$} & $\mathbf{a}$ & 0.28751 & 0.04436 & 6.48172 \\
\hline & b & 0.82118 & 0.10488 & 7.82993 \\
\hline \multicolumn{5}{|c|}{$r 2=0.939$} \\
\hline \multirow{2}{*}{$C 3$} & $\mathbf{a}$ & -0.47242 & 0.14715 & -3.21056 \\
\hline & b & 0.87933 & 0.24248 & 3.62637 \\
\hline \multicolumn{5}{|c|}{$\mathrm{r} 2=0.467$} \\
\hline \multirow{2}{*}{ C4 } & $\mathbf{a}$ & 0.11296 & 0.05406 & 2.0897 \\
\hline & b & 0.59863 & 0.10372 & 5.77186 \\
\hline \multicolumn{5}{|c|}{$\mathrm{r} 2=0.637$} \\
\hline \multirow{2}{*}{$C 5$} & $\mathbf{a}$ & 0.70259 & 0.02642 & 26.59207 \\
\hline & b & 0.65663 & 0.05315 & 12.3539 \\
\hline \multicolumn{5}{|c|}{$\mathrm{r} 2=0.437$} \\
\hline
\end{tabular}

Tabla 7. Modelación por categorías según parámetros ambientales.

\begin{tabular}{|c|c|c|c|c|}
\hline \multicolumn{5}{|c|}{$\log (P)=a+b \cdot \log (t)$} \\
\hline Categoría (Parámetros) & Variable & Coefficient & Standard error & t-ratio \\
\hline \multirow{2}{*}{$C 3$} & $\mathbf{a}$ & 0.51268 & 0.03737 & 13.71835 \\
\hline & b & 0.61791 & 0.07374 & 8.37974 \\
\hline \multicolumn{5}{|c|}{$\mathrm{r} 2=0.243$} \\
\hline \multirow{2}{*}{ C4 } & $\mathbf{a}$ & 0.82863 & 0.04656 & 17.79703 \\
\hline & b & 0.73075 & 0.10402 & 7.02502 \\
\hline \multicolumn{5}{|c|}{$\mathrm{r} 2=0.832$} \\
\hline \multirow{2}{*}{$C 5$} & $\mathbf{a}$ & 1.11527 & 0.05192 & 21.48252 \\
\hline & b & 0.59967 & 0.09924 & 6.04257 \\
\hline \multicolumn{5}{|c|}{$\mathrm{r} 2=0.753$} \\
\hline
\end{tabular}

Tabla 8. Modelación de todas las estaciones.

\begin{tabular}{|c|c|c|c|c|}
\hline \multicolumn{5}{|c|}{$\log (\mathrm{P})=\mathrm{a}+\mathrm{b} \cdot \log (\mathrm{t})$} \\
\hline \multirow{2}{*}{ Estaciones } & Variable & Coefficient & Standard error & \multirow{2}{*}{ t-ratio } \\
\hline \multirow{2}{*}{ Todas } & $\mathrm{a}$ & 0.56239 & 0.03585 & 15.68551 \\
\cline { 2 - 5 } & $\mathrm{b}$ & 0.62069 & 0.07099 & 8.74318 \\
\hline \multicolumn{5}{|c}{$\mathrm{r}^{2}=0.238$} \\
\hline
\end{tabular}


De la misma manera que con la ley potencial, se modelaron por separado cada una de las estaciones así como agrupadas en categorías, utilizando la ecuación 2. Tablas 9 - 15 .

Tabla 9. Coeficiente de correlación según el modelo propuesto.

\begin{tabular}{|c|c|c|c|c|c|}
\hline \multicolumn{6}{|c|}{$\log \mathrm{P}=\mathrm{a}+\mathrm{b} \cdot \log \mathrm{t}+\mathrm{k}_{1} \cdot\left[\mathrm{Cl}^{-}\right]+\mathrm{k}_{2} \cdot\left[\mathrm{SO}_{2}\right]+\mathrm{k}_{3} \cdot[\mathrm{TDH}]$} \\
\hline EST & $\mathrm{R}^{2}$ & Adj. R & EST & $\mathrm{R}^{2}$ & Adj. ${ }^{2}$ \\
\hline 1 & 0.9684 & 0.9578 & 21 & 0.9821 & 0.94645 \\
\hline 2 & 0.9944 & 0.98344 & 22 & 0.9994 & 0.99821 \\
\hline 3 & 0.9999 & 0.99971 & 23 & 0.9934 & 0.98033 \\
\hline 4 & 0.9839 & 0.95171 & 24 & 0.9945 & 0.98372 \\
\hline 5 & 0.9983 & 0.99489 & 25 & 0.8285 & 0.75995 \\
\hline 6 & 0.9164 & 0.88303 & 26 & 0.8685 & 0.34287 \\
\hline 7 & 0.9970 & 0.99115 & 27 & 0.2587 & -0.0376 \\
\hline 8 & 0.9951 & 0.98531 & 28 & 0.9994 & 0.99838 \\
\hline 9 & 0.9661 & 0.95262 & 29 & 0.9358 & 0.78673 \\
\hline 10 & 0.9871 & 0.96138 & 30 & 0.9876 & 0.96308 \\
\hline 11 & 0.9933 & 0.98017 & 31 & 0.9604 & 0.88129 \\
\hline 12 & 0.9865 & 0.95958 & 32 & 0.9554 & 0.86633 \\
\hline 13 & 0.9946 & 0.98394 & 33 & 0.9829 & 0.91483 \\
\hline 14 & 0.8064 & 0.41920 & 34 & 0.9530 & 0.85927 \\
\hline 15 & 0.9871 & 0.96146 & 35 & 0.8869 & 0.6636 \\
\hline 16 & 0.9818 & 0.84999 & 36 & 0.9399 & 0.81995 \\
\hline 17 & 0.9960 & 0.98036 & 37 & 0.9236 & 0.7709 \\
\hline 18 & 0.9858 & 0.95748 & 38 & 0.9615 & 0.88452 \\
\hline 19 & 0.7264 & 0.17943 & 39 & 0.2036 & -0.3951 \\
\hline 20 & 0.9280 & 0.78405 & & & \\
\hline
\end{tabular}

\section{Resultados estudios electroquímicos}

En la tabla 16 se muestran los resultados obtenidos al realizar las medidas de potencial de corrosión y Tafel en disoluciones individuales de $\mathrm{NaCl}$ y $\mathrm{Na}_{2} \mathrm{SO}_{4}$, mientras que en la tabla 17 se muestran los resultados obtenidos al realizar las medidas en disoluciones mezcla de $\mathrm{NaCl}$ y $\mathrm{Na}_{2} \mathrm{SO}_{4}$, manteniendo constante la concentración de $\mathrm{Na}_{2} \mathrm{SO}_{4}$ y variando la concentración de $\mathrm{NaCl}$, según se especificó en el apartado anterior. 
Tabla 10. Regresión con el modelo propuesto.

\begin{tabular}{|c|c|c|c|c|c|}
\hline \multicolumn{6}{|c|}{$\log \mathrm{P}=\mathrm{a}+\mathrm{b} \cdot \log \mathrm{t}+\mathrm{k}_{1} \cdot\left[\mathrm{Cl}^{-}\right]+\mathrm{k}_{2} \cdot\left[\mathrm{SO}_{2}\right]+\mathrm{k}_{3} \cdot[\mathrm{TDH}]$} \\
\hline EST & $\mathrm{a}$ & $\mathrm{b}$ & $\mathrm{k}_{1}$ & $\mathrm{k}_{2}$ & $\mathrm{k}_{3}$ \\
\hline 1 & -0.3118 & 3.6836 & 0.0109 & 0.5087 & $-6.19 \mathrm{E}-04$ \\
\hline 2 & 1.2356 & 0.5033 & -0.0103 & -0.3078 & $3.49 \mathrm{E}-05$ \\
\hline 3 & 0.0106 & 1.1019 & 0.0049 & 0.1276 & $-2.32 \mathrm{E}-05$ \\
\hline 4 & -0.3169 & 3.6987 & 0.0109 & 0.5095 & $-6.21 \mathrm{E}-04$ \\
\hline 5 & 1.7694 & 1.2602 & -0.0114 & -0.1233 & $-1.23 \mathrm{E}-01$ \\
\hline 6 & 2.7216 & -0.00007 & 0.0169 & 0.4447 & $-1.58 \mathrm{E}-04$ \\
\hline 7 & -1.3493 & 1.0849 & 0.0535 & 0.2545 & $-3.40 \mathrm{E}-06$ \\
\hline 8 & -0.2498 & 1.2558 & 0.0145 & 0.1580 & $-1.51 \mathrm{E}-05$ \\
\hline 9 & 1.0540 & -0.1573 & -0.0050 & -0.1908 & $4.641 \mathrm{E}-05$ \\
\hline 10 & 0.3991 & 1.4970 & 0.0288 & -1.9735 & $-5.60 \mathrm{E}-05$ \\
\hline 11 & 0.7484 & 0.5701 & 0.0030 & -0.8936 & $2.93 \mathrm{E}-05$ \\
\hline 12 & 0.0210 & 0.8204 & 0.0231 & -0.0799 & $5.21 \mathrm{E}-06$ \\
\hline 13 & 0.5631 & 0.4519 & -0.0004 & 0.0350 & $8.67 \mathrm{E}-06$ \\
\hline 14 & 0.0527 & 0.5117 & 0.0082 & -0.4585 & $4.11 \mathrm{E}-05$ \\
\hline 15 & 0.4866 & 0.4628 & 0.0011 & 0.0146 & $-1.27 \mathrm{E}-05$ \\
\hline 16 & -2.5645 & 0.0000 & 0.1298 & -1.2514 & $5.18 \mathrm{E}-05$ \\
\hline 17 & -4.0225 & 6.8387 & 0.4360 & -3.7372 & $-6.96 \mathrm{E}-04$ \\
\hline 18 & -1.3263 & 0.8978 & 0.0392 & 0.9627 & $3.35 \mathrm{E}-06$ \\
\hline 19 & 0.9044 & 1.0355 & 0.0301 & -6.614 & $1.62 \mathrm{E}-06$ \\
\hline 20 & 0.1195 & 0.4917 & 0.0597 & -10.33 & $9.86 \mathrm{E}-06$ \\
\hline 21 & 0.5432 & 0.7616 & 0.0085 & -0.5758 & $8.81 \mathrm{E}-06$ \\
\hline 22 & 1.2384 & 0.7165 & 0.0001 & -0.0092 & $-2.13 E-06$ \\
\hline 23 & -0.1012 & 1.0304 & 0.0265 & -0.0176 & $-1.00 \mathrm{E}-05$ \\
\hline 24 & -0.8426 & 0.7138 & 0.0118 & 0.3331 & $5.20 \mathrm{E}-06$ \\
\hline 25 & 5.9727 & -4.4432 & -0.0663 & -0.3378 & $7.09 \mathrm{E}-05$ \\
\hline 26 & 0.1745 & 2.0229 & -0.0280 & -5.1021 & $-3.24 \mathrm{E}-05$ \\
\hline 27 & 3.3002 & 1.0715 & -0.2512 & 0.1204 & $-6.26 \mathrm{E}-05$ \\
\hline 28 & -0.6118 & 1.0422 & 0.0455 & 1.6098 & $-8.89 \mathrm{E}-06$ \\
\hline 29 & -0.5598 & 0.0000 & 0.0019 & 0.4299 & $7.85 \mathrm{E}-05$ \\
\hline 30 & 0.1258 & 0.5893 & 0.0126 & -0.7466 & $-1.27 \mathrm{E}-05$ \\
\hline 31 & 0.5343 & 0.8099 & 0.0393 & -2.3208 & $4.92 \mathrm{E}-06$ \\
\hline 32 & 1.1890 & -0.0158 & -0.0199 & -7.2457 & $1.97 \mathrm{E}-05$ \\
\hline 33 & -0.2636 & 0.1248 & -0.0002 & 0.3607 & $6.99 \mathrm{E}-05$ \\
\hline 34 & 0.2898 & 0.8125 & 0.0118 & 0.0241 & $2.09 \mathrm{E}-05$ \\
\hline 35 & -7.7504 & 1.8154 & 0.4760 & 0.0000 & $4.43 \mathrm{E}-06$ \\
\hline 36 & 0.0409 & 0.6537 & -0.0061 & 0.0345 & $1.32 \mathrm{E}-06$ \\
\hline 37 & -1.267 & 1.5645 & 0.0583 & 0.3041 & $-4.40 \mathrm{E}-05$ \\
\hline 38 & 0.6357 & 0.4755 & -0.0263 & 0.1142 & $4.20 \mathrm{E}-06$ \\
\hline 39 & -2.2035 & 0.0000 & 0.1407 & 0.0000 & 2.3523 \\
\hline
\end{tabular}


En la Fig. 3 se representa la variación de la resistencia de polarización $\left(R_{p}\right)$ de cada medio contaminante frente a la variación en la concentración de cada uno de ellos y en la Fig. 4 esta variación con la acción conjunta de los dos, manteniendo fija la de $\mathrm{Na}_{2} \mathrm{SO}_{4}$ y variando la de $\mathrm{NaCl}$.

En las Fig. 5 a la 7 se muestran las gráficas correspondientes a los Tafel obtenidos. En la figura 8 se representa una de las gráficas de polarización a elevados niveles de potencial de barrido.

Tabla 11. Coeficiente de correlación del modelo propuesto por categorías según pérdida de peso.

\begin{tabular}{|c|c|c|}
\hline \multicolumn{2}{|c|}{$\log \mathrm{P}=\mathrm{a}+\mathrm{b} \cdot \log \mathrm{t}+\mathrm{k}_{1} \cdot\left[\mathrm{Cl}^{-}\right]+\mathrm{k}_{2} \cdot\left[\mathrm{SO}_{2}\right]+\mathrm{k}_{3} \cdot[\mathrm{TDH}]$} \\
\hline Categoría (perdida peso) & $\mathrm{R}^{2}$ & Adj. $\mathrm{R}^{2}$ \\
\hline $\mathrm{C} 1$ & 0.98297 & 0.91483 \\
\hline $\mathrm{C} 3$ & 0.58231 & 0.44308 \\
\hline $\mathrm{C} 4$ & 0.69899 & 0.62373 \\
\hline $\mathrm{C} 5$ & 0.53221 & 0.52257 \\
\hline
\end{tabular}

Tabla 12. Regresión con el modelo propuesto por categorías según pérdida de peso.

\begin{tabular}{|c|c|c|c|c|c|}
\hline \multicolumn{6}{|c|}{$\log \mathrm{P}=\mathrm{a}+\mathrm{b} \cdot \log \mathrm{t}+\mathrm{k}_{1} \cdot\left[\mathrm{Cl}^{-}\right]+\mathrm{k}_{2} \cdot\left[\mathrm{SO}_{2}\right]+\mathrm{k}_{3} \cdot[\mathrm{TDH}]$} \\
\hline Categoría (perdida peso) & $\mathrm{a}$ & $\mathrm{b}$ & $\mathrm{k}_{1}$ & $\mathrm{k}_{2}$ & $\mathrm{k}_{3}$ \\
\hline $\mathrm{C} 1$ & -0.2636 & 0.1248 & -0.00024 & 0.3607 & $6.991 \mathrm{E}-05$ \\
\hline $\mathrm{C} 3$ & 0.7315 & 0.3778 & -0.11412 & 2.0756 & $8.724 \mathrm{E}-06$ \\
\hline $\mathrm{C} 4$ & 0.1308 & 0.5118 & 0.00119 & -0.1909 & $6.708 \mathrm{E}-06$ \\
\hline $\mathrm{C} 5$ & 0.6517 & 0.6289 & 0.00087 & -0.0076 & $2.539 \mathrm{E}-06$ \\
\hline
\end{tabular}

Tabla 13. Coeficiente de correlación del modelo propuesto por categorías según parámetros atmosféricos.

\begin{tabular}{|c|c|c|}
\hline \multicolumn{2}{|c|}{$\log \mathrm{P}=\mathrm{a}+\mathrm{b} \cdot \log \mathrm{t}+\mathrm{k}_{1} \cdot\left[\mathrm{Cl}^{-}\right]+\mathrm{k}_{2} \cdot\left[\mathrm{SO}_{2}\right]+\mathrm{k}_{3} \cdot[\mathrm{TDH}]$} \\
\hline Categoría (parámetros) & $\mathrm{R}^{2}$ & Adj. $\mathrm{R}^{2}$ \\
\hline C3 & 0.34881 & 0.33675 \\
\hline C4 & 0.98825 & 0.98153 \\
\hline C5 & 0.94735 & 0.92394 \\
\hline
\end{tabular}


Tabla 14. Regresión con el modelo propuesto por categorías según parámetros atmosféricos.

\begin{tabular}{|c|c|c|c|c|c|}
\hline \multicolumn{6}{|c|}{$\log \mathrm{P}=\mathrm{a}+\mathrm{b} \cdot \log \mathrm{t}+\mathrm{k}_{1} \cdot\left[\mathrm{Cl}^{-}\right]+\mathrm{k}_{2} \cdot\left[\mathrm{SO}_{2}\right]+\mathrm{k}_{3} \cdot[\mathrm{TDH}]$} \\
\hline Categoría (parámetros) & $\mathrm{a}$ & $\mathrm{b}$ & $\mathrm{k}_{1}$ & $\mathrm{k}_{2}$ & $\mathrm{k}_{3}$ \\
\hline C3 & 0.33548 & 0.78535 & 0.00524 & $-6 \mathrm{E}-05$ & $-8.491 \mathrm{E}-06$ \\
\hline C4 & 0.67767 & 0.34829 & -0.00067 & 0.0286 & $1.051 \mathrm{E}-05$ \\
\hline C5 & 1.02366 & 0.70095 & 0.000765 & -0.0361 & $-6.672 \mathrm{E}-07$ \\
\hline
\end{tabular}

Tabla 15. Valores obtenidos al modelar el conjunto de las estaciones.

\begin{tabular}{|c|c|c|c|}
\hline \multicolumn{3}{|l}{$\log \mathrm{P}=\mathrm{a}+\mathrm{b} \cdot \log \mathrm{t}+\mathrm{k}_{1} \cdot\left[\mathrm{Cl}^{-}\right]+\mathrm{k}_{2} \cdot\left[\mathrm{SO}_{2}\right]+\mathrm{k}_{3} \cdot[\mathrm{TDH}]$} \\
\hline Variable & Coefficient & Standard error & t-ratio \\
\hline $\mathrm{a}$ & 0.33548 & 0.05518 & 6.07958 \\
\hline $\mathrm{b}$ & 0.78535 & 0.11144 & 7.0472 \\
\hline $\mathrm{k}_{1}$ & 0.00524 & 0.00107 & 4.88731 \\
\hline $\mathrm{k}_{2}$ & $-5.61772 \cdot 10^{-5}$ & 0.00793 & -0.00709 \\
\hline $\mathrm{k}_{3}$ & $-8.49086 \cdot 10^{-6}$ & $-5.98641 \cdot 10^{-6}$ & -1.41836 \\
\hline \multicolumn{4}{|c}{$\mathrm{r}^{2}=0.349$} \\
\hline
\end{tabular}

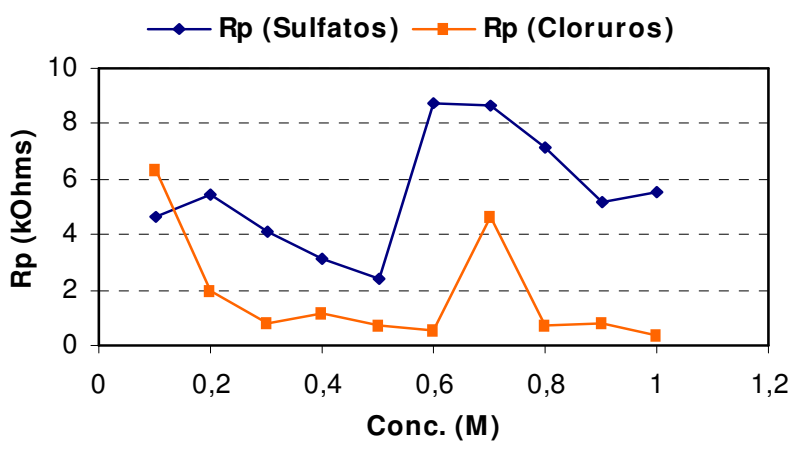

Figura 3. Variación de la $\mathrm{R}_{\mathrm{p}}$ con la concentración de cloruros y sulfatos.

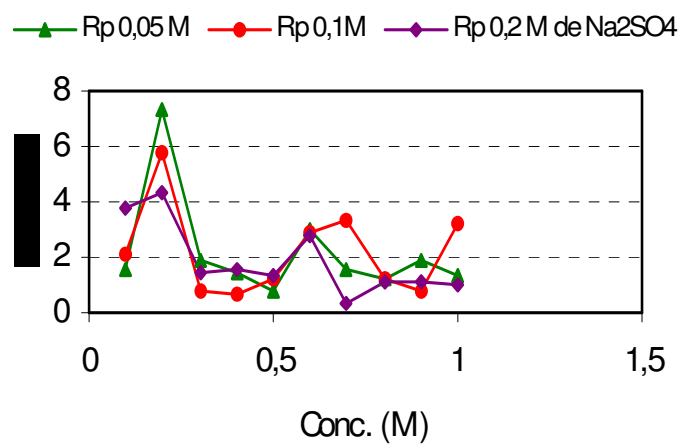

Figura 4. Variación de la $\mathrm{R}_{\mathrm{p}}$ en disolución de $\mathrm{NaCl}$ frente a concentración fija de $\mathrm{Na}_{2} \mathrm{SO}_{4}$. 
Tabla 16. Valores de Tafel y $\mathrm{E}_{\text {corr }}$ en disoluciones de $\mathrm{NaCl}$ y $\mathrm{Na}_{2} \mathrm{SO}_{4}$.

\begin{tabular}{|c|c|c|c|}
\hline Conc. & Parámetro & $\mathrm{NaCl}$ (Valores medios) & $\mathrm{Na}_{2} \mathrm{SO}_{4}$ (Valores medios) \\
\hline \multirow{3}{*}{$0.1 \mathrm{M}$} & $\mathrm{i}_{0}[\mu \mathrm{A}]$ & 2.513 & 3.106 \\
\hline & $\mathrm{E}_{\text {corr }}[\mathrm{mV}]$ & -25.5 & -47.98 \\
\hline & $\mathrm{R}_{\mathrm{p}}[\mathrm{kOhm}]$ & 6.325 & 4.656 \\
\hline \multirow{3}{*}{$0.2 \mathrm{M}$} & $\mathrm{i}_{0}[\mu \mathrm{A}]$ & 2.631 & 3.111 \\
\hline & $\mathrm{E}_{\text {corr }}[\mathrm{mV}]$ & -227.3 & -37.15 \\
\hline & $\mathrm{R}_{\mathrm{p}}[\mathrm{kOhm}]$ & 1.935 & 5.405 \\
\hline \multirow{3}{*}{$0.3 \mathrm{M}$} & $\mathrm{i}_{0}[\mu \mathrm{A}]$ & 12.55 & 0.308 \\
\hline & $\mathrm{E}_{\text {corr }}[\mathrm{mV}]$ & -97.56 & -40.23 \\
\hline & $\mathrm{R}_{\mathrm{p}}[\mathrm{kOhm}]$ & 0.808 & 4.117 \\
\hline \multirow{3}{*}{$0.4 \mathrm{M}$} & $\mathrm{i}_{0}[\mu \mathrm{A}]$ & 16.62 & 5.122 \\
\hline & $\mathrm{E}_{\text {corr }}[\mathrm{mV}]$ & -218.5 & -53.07 \\
\hline & $\mathrm{R}_{\mathrm{p}}[\mathrm{kOhm}]$ & 1.152 & 3.128 \\
\hline \multirow{3}{*}{$0.5 \mathrm{M}$} & $\mathrm{i}_{0}[\mu \mathrm{A}]$ & 40.31 & 7.1 \\
\hline & $\mathrm{E}_{\text {corr }}[\mathrm{mV}]$ & -120.9 & -27.43 \\
\hline & $\mathrm{R}_{\mathrm{p}}[\mathrm{kOhm}]$ & 0.756 & 2.430 \\
\hline \multirow{3}{*}{$0.6 \mathrm{M}$} & $\mathrm{i}_{0}[\mu \mathrm{A}]$ & 5.44 & 0.172 \\
\hline & $\mathrm{E}_{\text {corr }}[\mathrm{mV}]$ & -258 & -5.532 \\
\hline & $\mathrm{R}_{\mathrm{p}}[\mathrm{kOhm}]$ & 0.533 & 8.737 \\
\hline \multirow{3}{*}{$0.7 \mathrm{M}$} & $\mathrm{i}_{0}[\mu \mathrm{A}]$ & 3.632 & 4.471 \\
\hline & $\mathrm{E}_{\text {corr }}[\mathrm{mV}]$ & -184.3 & 18.11 \\
\hline & $\mathrm{R}_{\mathrm{p}}[\mathrm{kOhm}]$ & 4.032 & 8.686 \\
\hline \multirow{3}{*}{$0.8 \mathrm{M}$} & $\mathrm{i}_{0}[\mu \mathrm{A}]$ & 9.7 & 13.63 \\
\hline & $\mathrm{E}_{\mathrm{corr}}[\mathrm{mV}]$ & -265.1 & -4.544 \\
\hline & $\mathrm{R}_{\mathrm{p}}[\mathrm{kOhm}]$ & 0.736 & 7.166 \\
\hline \multirow{3}{*}{$0.9 \mathrm{M}$} & $\mathrm{i}_{0}[\mu \mathrm{A}]$ & 26.37 & 4.686 \\
\hline & $\mathrm{E}_{\text {corr }}[\mathrm{mV}]$ & -239 & -17.74 \\
\hline & $\mathrm{R}_{\mathrm{p}}[\mathrm{kOhm}]$ & 0.768 & 5.139 \\
\hline \multirow{3}{*}{$1 \mathrm{M}$} & $\mathrm{i}_{0}[\mu \mathrm{A}]$ & 44.02 & 1.424 \\
\hline & $\mathrm{E}_{\text {corr }}[\mathrm{mV}]$ & -258.9 & -49.88 \\
\hline & $\mathrm{R}_{\mathrm{p}}[\mathrm{kOhm}]$ & 0.362 & 6.778 \\
\hline
\end{tabular}

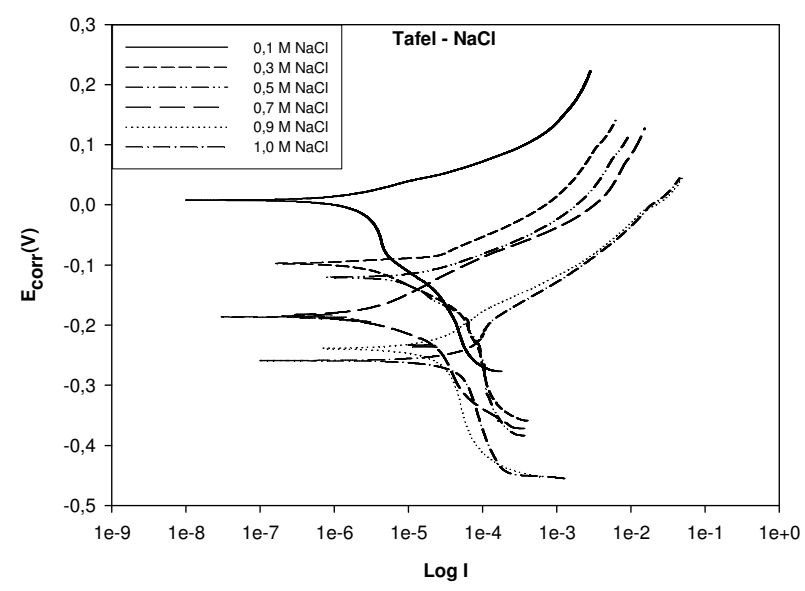

Figura 5. Diagrama Tafel en $\mathrm{NaCl} 0.1-1 \mathrm{M}$. 
Tabla 17. Resultados de Tafel y $\mathrm{E}_{\text {corr }}$ en disoluciones mezcla de $\mathrm{NaCl}$ y $\mathrm{Na}_{2} \mathrm{SO}_{4}$.

\begin{tabular}{|c|c|c|c|c|}
\hline \multirow{2}{*}{ Conc. $\mathrm{NaCl}$} & \multirow{2}{*}{ Parámetro } & \multicolumn{3}{|c|}{$\mathrm{NaCl}+$} \\
\hline & & $\mathrm{Na}_{2} \mathrm{SO}_{4} 0.05 \mathrm{M}$ & $\mathrm{Na}_{2} \mathrm{SO}_{4} 0.1 \mathrm{M}$ & $\mathrm{Na}_{2} \mathrm{SO}_{4} 0.2 \mathrm{M}$ \\
\hline \multirow{3}{*}{$0,1 \mathrm{M}$} & $\mathrm{i}_{0}[\mu \mathrm{A}]$ & 10.09 & 8.39 & 4.317 \\
\hline & $\mathrm{E}_{\text {corr }}[\mathrm{mV}]$ & -112.1 & -107.7 & -108.1 \\
\hline & $\mathrm{R}_{\mathrm{p}}[\mathrm{kOhm}]$ & 1.534 & 2.098 & 3.807 \\
\hline \multirow{3}{*}{$0,2 \mathrm{M}$} & $\mathrm{i}_{0}[\mu \mathrm{A}]$ & 1.698 & 4.268 & 3.827 \\
\hline & $\mathrm{E}_{\text {corr }}[\mathrm{mV}]$ & -103.4 & -160.9 & -140.5 \\
\hline & $\mathrm{R}_{\mathrm{p}}[\mathrm{kOhm}]$ & 7.297 & 5.833 & 4.359 \\
\hline \multirow{3}{*}{$0,3 \mathrm{M}$} & $\mathrm{i}_{0}[\mu \mathrm{A}]$ & 2.513 & 23.99 & 15.1 \\
\hline & $\mathrm{E}_{\text {corr }}[\mathrm{mV}]$ & -283.9 & -148.7 & -154 \\
\hline & $\mathrm{R}_{\mathrm{p}}[\mathrm{kOhm}]$ & 1.884 & 0.82 & 1.424 \\
\hline \multirow{3}{*}{$0,4 \mathrm{M}$} & $\mathrm{i}_{0}[\mu \mathrm{A}]$ & 21.98 & 43.44 & 3.578 \\
\hline & $\mathrm{E}_{\text {corr }}[\mathrm{mV}]$ & -159.8 & -166.7 & -277.4 \\
\hline & $\mathrm{R}_{\mathrm{p}}[\mathrm{kOhm}]$ & 1.423 & 0.635 & 1.513 \\
\hline \multirow{3}{*}{$0,5 \mathrm{M}$} & $\mathrm{i}_{0}[\mu \mathrm{A}]$ & 0.781 & 18.18 & 5.245 \\
\hline & $\mathrm{E}_{\text {corr }}[\mathrm{mV}]$ & -184.9 & -176 & -280.6 \\
\hline & $\mathrm{R}_{\mathrm{p}}[\mathrm{kOhm}]$ & 8.975 & 1.174 & 1.382 \\
\hline \multirow{3}{*}{$0,6 \mathrm{M}$} & $i_{0}[\mu \mathrm{A}]$ & 2.542 & 4.196 & 1.503 \\
\hline & $\mathrm{E}_{\text {corr }}[\mathrm{mV}]$ & -260.4 & -242.4 & -255.2 \\
\hline & $\mathrm{R}_{\mathrm{p}}[\mathrm{kOhm}]$ & 3.05 & 2.932 & 2.802 \\
\hline \multirow{3}{*}{$0,7 \mathrm{M}$} & $\mathrm{i}_{0}[\mu \mathrm{A}]$ & 2.680 & 3.129 & 15.61 \\
\hline & $\mathrm{E}_{\text {corr }}[\mathrm{mV}]$ & -283.9 & -253.4 & -279.2 \\
\hline & $\mathrm{R}_{\mathrm{p}}[\mathrm{kOhm}]$ & 1.528 & 3.345 & 0.314 \\
\hline \multirow{3}{*}{$0,8 \mathrm{M}$} & $\mathrm{i}_{0}[\mu \mathrm{A}]$ & 1.547 & 0.848 & 3.234 \\
\hline & $\mathrm{E}_{\text {corr }}[\mathrm{mV}]$ & -281.3 & -281.5 & -280.9 \\
\hline & $\mathrm{R}_{\mathrm{p}}[\mathrm{kOhm}]$ & 1.241 & 1.272 & 1.124 \\
\hline \multirow{3}{*}{$0,9 \mathrm{M}$} & $\mathrm{i}_{0}[\mu \mathrm{A}]$ & 15.69 & 16.74 & 1.888 \\
\hline & $\mathrm{E}_{\text {corr }}[\mathrm{mV}]$ & -216.5 & -264 & -289.8 \\
\hline & $\mathrm{R}_{\mathrm{p}}[\mathrm{kOhm}]$ & 1.845 & 0.815 & 1.061 \\
\hline \multirow{3}{*}{$1 \mathrm{M}$} & $\mathrm{i}_{0}[\mu \mathrm{A}]$ & 0.622 & 0.678 & 9.909 \\
\hline & $\mathrm{E}_{\text {corr }}[\mathrm{mV}]$ & -277.1 & -250.6 & -287 \\
\hline & $\mathrm{R}_{\mathrm{p}}[\mathrm{kOhm}]$ & 1.286 & 3.197 & 1.041 \\
\hline
\end{tabular}

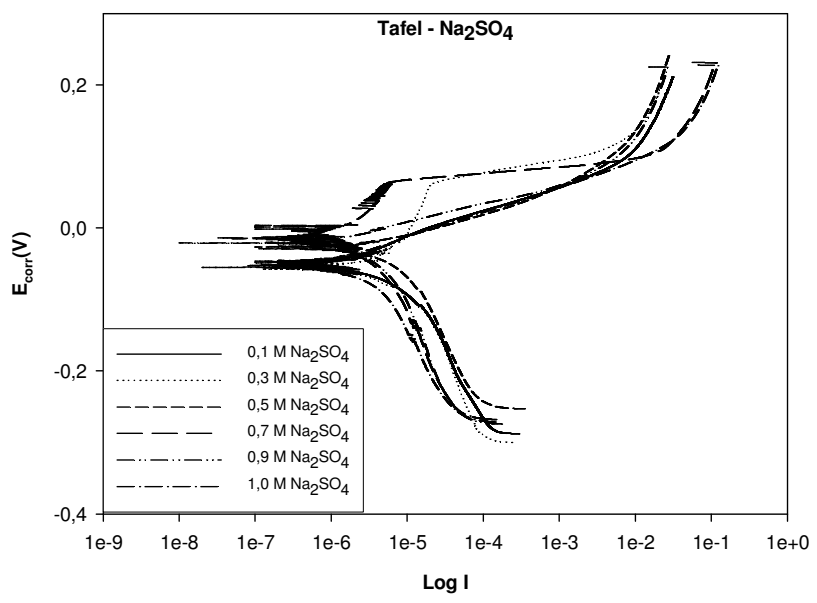

Figura 6. Diagrama Tafel en $\mathrm{Na}_{2} \mathrm{SO}_{4} 0.1-1 \mathrm{M}$. 


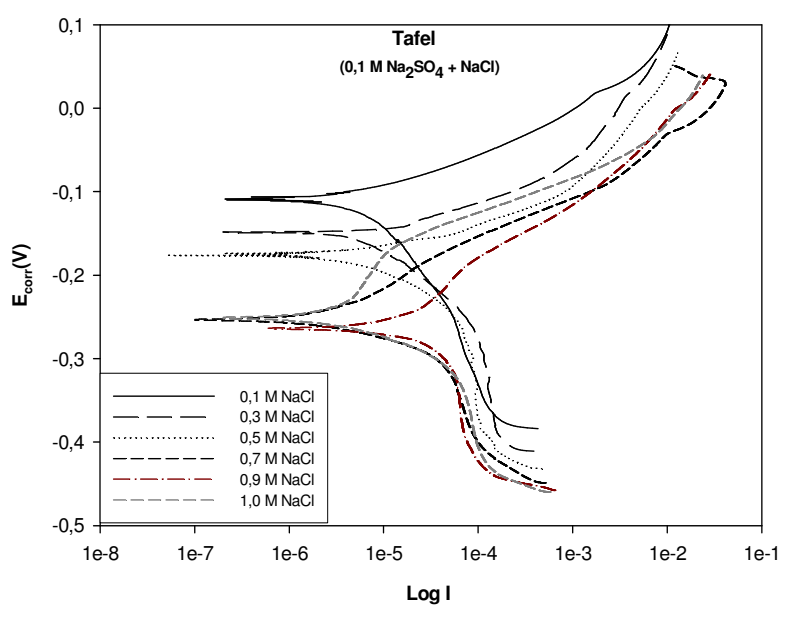

Figura 7. Diagrama Tafel en $\mathrm{NaCl}+\mathrm{Na}_{2} \mathrm{SO}_{4}$.

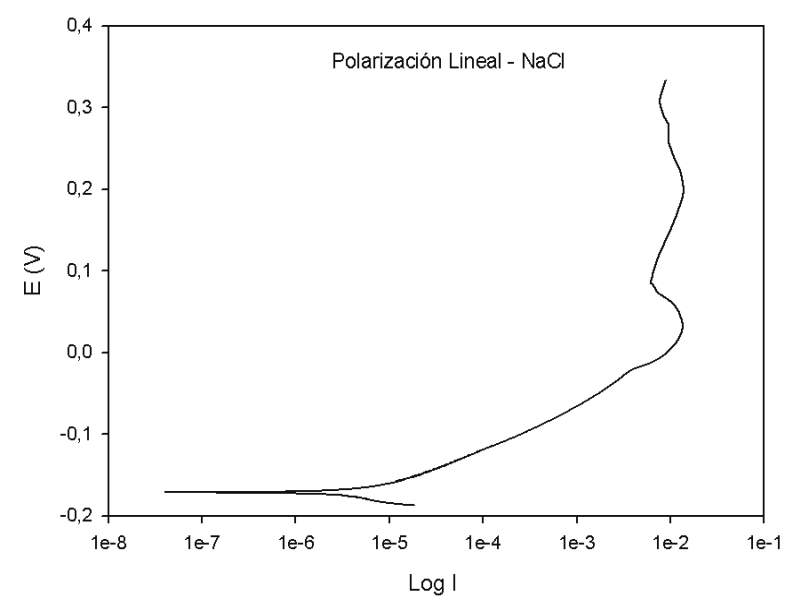

Figura 8. Polarización lineal en $\mathrm{NaCl} 0.4 \mathrm{M}$.

\section{Discusión de los resultados \\ Corrosión atmosférica}

El cobre sufre un aumento considerable en su velocidad de corrosión al entrar en contacto con atmósferas conteniendo cloruros y $\mathrm{SO}_{2}$, siendo el cloruro un factor más influyente en cuanto al aumento cuantitativo de la velocidad. De forma general se observa que la pérdida de peso aumenta con el tiempo de exposición, mientras que la velocidad de corrosión disminuye, debido al efecto protector de la pátina que se forma sobre la placa de cobre. Por otra parte se ha constatado el tipo de corrosión generalizada producida en las probetas utilizadas para el estudio, descartado en estos ambientes posibles procesos de corrosión por picadura que distorsionen los niveles de pérdidas de peso observados.

Se encuentra que para la isla de Fuerteventura y la isla de Lanzarote, las categorías de corrosividad obtenidas por pérdida de peso son en todos los casos $\mathrm{C} 5$, es decir, obtenemos la máxima categoría para todas las estaciones de dos 
islas. Si observamos el intervalo correspondiente a la categoría C5 para el cobre, según se recoge en la norma ISO 9223, este intervalo es $25<\mathrm{r}_{\text {corr }} \leq 50$ $\mathrm{g} /\left(\mathrm{m}^{2} \cdot\right.$ año $),\left(3.04<\mathrm{r}_{\text {corr }} \leq 6.08 \mu / \mathrm{año}\right)$.

En la isla de Fuerteventura, en cuatro de las seis estaciones se superó la barrera de $50 \mathrm{~g} /\left(\mathrm{m}^{2} \cdot a n ̃ o\right)$, llegando incluso casi a duplicarse, como es el caso de la estación $3\left(84 \mathrm{~g} /\left(\mathrm{m}^{2} \cdot a n ̃ o\right)\right)$. En la isla de Lanzarote se registraron en 3 estaciones valores iguales o superiores a $50 \mathrm{~g} /\left(\mathrm{m}^{2}\right.$ año), concretamente en las estaciones 8,9 y 11 , mientras que en la estación 7 se registraron valores de $32 \mathrm{~g} /\left(\mathrm{m}^{2} \cdot a n ̃ o\right)$, (Fig. 9 y 10$)$.

De un análisis de los resultados obtenidos en las estaciones de la isla Gran Canaria se desprende que 18 de las 27 estaciones presentan una categoría de corrosividad C5, 5 presentan una categoría de corrosividad C4, una sola estación (estación de Tejeda) categoría C3 y con categoría de corrosividad C2 tres estaciones 16 (Sta. Brígida), 26 (San Mateo) y 35 (Teror).

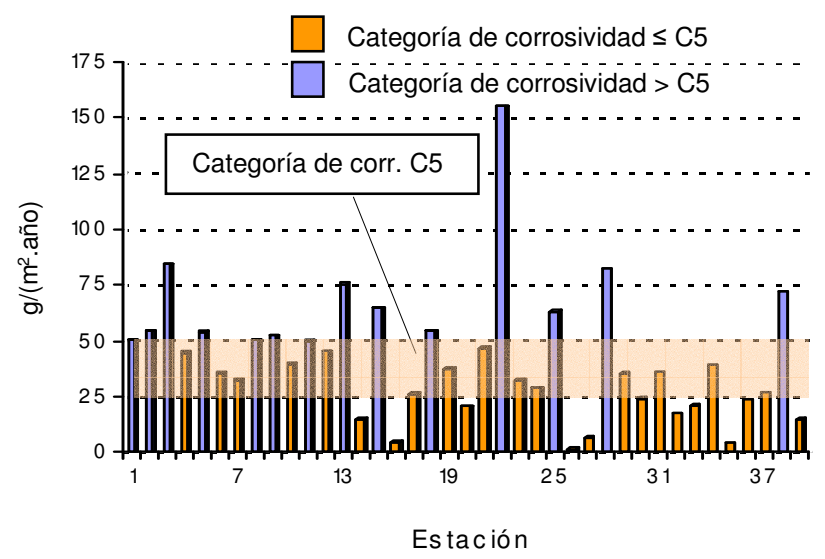

Figura 9. Distribución de categorías según velocidad de corrosión en el primer año.

En resumen, el $76.9 \%$ de las estaciones presenta categoría de corrosión C5, mientras que el $43.3 \%$ supera el límite superior impuesto en la norma. Esto pone de manifiesto que la norma no contempla casi la mitad de los casos encontrados en la provincia de Las Palmas, ya que más del $43 \%$ de los mismos superan el límite superior, con un caso excepcional, correspondiente a la estación 22 (Taliarte, Gran Canaria), donde la velocidad de corrosión triplica el valor máximo, con una velocidad de corrosión de $155 \mathrm{~g} /\left(\mathrm{m}^{2} \cdot a n ̃ o\right)$. Este hecho pone de manifiesto que la norma ISO 9223 no recoge estos casos en su clasificación de categorías de corrosividad. Si bien es cierto que las atmósferas predominantes en la mayoría de las estaciones ubicadas en Gran Canaria son marinas, no todas poseen el mismo grado de severidad, ya que los niveles de cloruros fluctúan de unos casos a otros. Esto pone de manifiesto que han de intervenir otros factores que influyen notablemente en la velocidad de corrosión y que no son contemplados por la norma. 


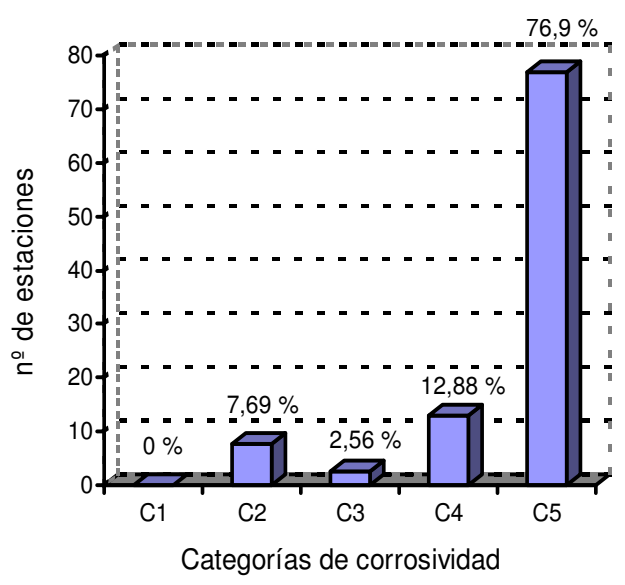

Figura 10. Porcentaje de estaciones por categorías.

\section{Modelación matemática}

De la modelación independiente de cada estación se observa que 28 de las 39 estaciones (un $71.8 \%$ de las estaciones) se ajusta a la ley bilogarítmica $\left(r^{2}>0.8\right)$, presentando 22 de ellas un índice de regresión superior a 0.9 , y más concretamente 7 presentan un índice de regresión superior a 0.95 . Se deduce, por tanto, que las estaciones en general se ajustan en buena medida a la ley bilogarítmica. De hecho, sólo 7 estaciones presentan un índice de regresión comprendido entre 0.8 y $0.9,6$ estaciones se sitúan entre 0.7 y 0.8 , situándose 4 estaciones por debajo de 0.7. A primera vista se observa que existe una concordancia entre la evolución de la corrosión del cobre y el tiempo de exposición, verificándose que la ley bilogarítmica se ajusta muy bien a la hora de intentar correlacionar los datos de pérdida de peso con el tiempo de exposición.

En cuanto a la modelación con la ecuación 2 para cada estación, cuyos resultados se detallan en la tabla 9 , observamos que un $89.74 \%$ de las estaciones se ajusta a la ecuación propuesta $\left(\mathrm{r}^{2}>0.8\right)$, presentando 32 de 39 un índice de regresión superior a 0.9 , y más concretamente 20 presentan un índice de regresión superior a 0.98 . Se consigue, por tanto, un modelo al que se ajusta de forma bastante buena el comportamiento del proceso corrosivo en cada estación.

Del resto de estaciones sólo una tiene un valor de $\mathrm{r}^{2}$ inferior a $0.8(0.72)$, mientras que las tres restantes carecen de datos suficientes para realizar la regresión.

A la hora de estudiar el comportamiento estadístico para las estaciones con una misma categoría de corrosividad encontramos que con el modelo propuesto obtenemos un mejor ajuste que con la ley bilogarítmica, resultando valores de $\mathrm{r}^{2}$

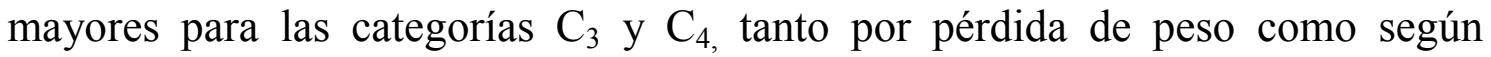
parámetros ambientales (tablas $6,7,11$ y 13).

Analizando el conjunto de las estaciones usando la ley potencial se obtiene un $\mathrm{r}^{2}$ de 0.238 mientras que con el modelo propuesto obtenemos un valor de 0.346 que, aunque mejora, está bastante alejado de la unidad debido al elevado número de estaciones y diversidad de factores de influencia. 


\section{Estudios electroquímicos}

El efecto que ejercen los iones cloruro y sulfato en la corrosión del cobre ha sido poco estudiado desde el punto de vista electroquímico en cuanto a su relación con la corrosión atmosférica.

Analizando las gráficas de potencial de corrosión $\mathrm{E}_{\text {corr }}$ en circuito abierto se observa un comportamiento muy estable del cobre, con un tiempo limite de estabilización del potencial de 1800 segundos. De los resultados expuestos en las tablas 16 y 17 se puede observar que no existe una variación lineal del potencial de corrosión a medida que aumentamos la concentración del agente agresivo, sea $\mathrm{NaCl}$ o $\mathrm{Na}_{2} \mathrm{SO}_{4}$. El mismo comportamiento ha sido observado en las variables $\mathrm{R}_{\mathrm{p}}$ e $\mathrm{I}_{\text {corr }}$ donde el perfil obtenido no es uniforme. Si tenemos en cuenta que todas las probetas han sido sometidas a un mismo periodo de inmersión inicial se descarta el hecho de que estas oscilaciones puedan ser debidas a la formación de una capa de productos de corrosión más o menos compacta.

En las Fig. 5, 6 y 7 se observa que todas las gráficas Tafel presentan el mismo perfil para la disolución de iones cloruro, sulfato y mezcla de los dos agentes agresivos. En la figura 8 se observa que polarizando el metal a elevados rangos de potencial no se observan incrementos de la densidad de corriente que pudieran asociarse a fenómenos de corrosión por picadura, a pesar de usar concentraciones de $\mathrm{NaCl}$ muy superiores a las dadas en el medio natural estudiado.

En las Fig. 3 y 4 se muestra la resistencia a la corrosión en cada medio agresivo y se establece una comparativa entre la evolución de la $R_{p}$ en cloruros y sulfatos. Se observa que la resistencia la corrosión va descendiendo a medida que aumenta la concentración tanto de iones cloruro como sulfatos, alcanzando su valor máximo de la $\mathrm{R}_{\mathrm{p}}$ a una concentración de $0.6 \mathrm{M}$ de iones sulfato y de $0.7 \mathrm{M}$ iones cloruro respectivamente. Una concentración de $0.6 \mathrm{M}$ se corresponde con agua de mar de $35 \mathrm{~g} / \mathrm{L}$. En esta zona tiene lugar la reacción donde se forma $\mathrm{Cu}_{2} \mathrm{O}$ que precipita, impidiendo de forma momentánea el proceso corrosivo sobre la superficie metálica, pero a medida que la concentración de contaminantes aumenta se va disolviendo dando lugar a compuestos más solubles que favorecen el proceso corrosivo.

En todo los casos, la intensidad de corrosión obtenida para iones cloruro es superior a la obtenida para iones sulfatos, proceso relacionado con la formación de atacamita que es un producto de corrosión 100 veces mas soluble que el sulfato de cobre [13].

En el caso de mezcla de contaminantes se observa un efecto sinérgico en cuanto al aumento de la $R_{p}$ al adicionarle pequeñas concentraciones de sulfatos a la disolución de cloruros. La variación en la evolución del perfil de la $R_{p}$ en los tres casos examinados sigue la misma tendencia que en el caso de las disoluciones de cloruro y sulfato por separado. Los valores de $\mathrm{I}_{\text {corr }}$ se asemejan más a los obtenidos para el caso de los iones cloruro por separado, lo cual nos indica que el ión sulfato ejerce una pequeña influencia en el proceso corrosivo. Se observa que para concentraciones bajas de $\mathrm{NaCl}$, el valor de $\mathrm{R}_{\mathrm{p}}$ observado es inferior al valor observado por sulfato sólo (Fig. 4). A partir de la concentración de $0.5 \mathrm{M} \mathrm{el}$ valor de $\mathrm{R}_{\mathrm{p}}$ es superior al valor del cloruro sólo, pero mucho menor que el valor obtenido por el sulfato sólo. Esto nos lleva a determinar que el ión cloruro afecta 
a la estructura de los productos de corrosión en mayor medida que lo hace el ión sulfato.

\section{Conclusiones}

- El cobre presenta unas velocidades de corrosión elevadas en la provincia de Las Palmas, con valores superiores al límite superior establecido para la categoría C5 en la norma ISO 9223. E1 76.9\% de las estaciones presentan una categoría de corrosividad C5. Esto es debido a los elevados niveles registrados de iones cloruro y a la humedad reinante en las distintas estaciones. La concentración de $\mathrm{SO}_{2}$ no se presenta como un factor decisivo para explicar las elevadas velocidades de corrosión en las distintas estaciones. Este proceso aumenta en las islas más occidentales por la presencia de polvo y arenisca los cuáles actúan a modo de granalla sobre la superficie metálica al ser arrastrados por el viento.

- El principal contaminante de las islas Canarias es el ión cloruro y también representa la variable mas significativa del análisis estadístico, bien sea de forma inversa, logaritmica o lineal.

- La gran variedad registrada en los niveles de agentes agresivos hace que una ecuación donde sólo se correlacionan las velocidades de corrosión con el tiempo de exposición sea menos representativa.

- De los estudios electroquímicos efectuados se desprende que, a concentraciones iguales de agentes agresivos, el ión cloruro provoca intensidades mayores de corrosión que el ión sulfato, lo que se traduce en una mayor velocidad de corrosión.

- De estos ensayos también se observa que el ión cloruro ejerce un efecto más acusado en la velocidad de corrosión respecto al ión sulfato.

- A bajas concentraciones de iones cloruro y sulfato de manera conjunta se produce un efecto de pasivasión, poniéndose de manifiesto el aumento de la $\mathrm{R}_{\mathrm{p}}$.

\section{Modelación de la corrosión atmosférica del cobre en la provincia de Las Palmas. \\ Estudios mediante técnicas clásicas y electroquímicas}

\section{Resumen}

La simulación de la acción de atmósferas naturales sobre metales empleando técnicas electroquímicas conjuntamente con ensayos acelerados presenta unas características que lo hacen muy interesante desde el punto de vista del estudio y predicción de la corrosión, ya que nos permitirá prever el comportamiento de un metal expuesto en un ambiente determinado sin necesidad de realizar ensayos de campo, con el consiguiente ahorro económico y de tiempo. Este estudio tiene la finalidad de correlacionar la velocidad de corrosión atmosférica del cobre obtenida mediante exposición atmosférica en estaciones en la provincia de Las Palmas con las distintas variables que intervienen en el proceso corrosivo. De la misma manera para la determinación del modelo matemático que mejor se ajuste a los resultados obtenidos experimentalmente y tenga 
en cuenta los parámetros de mayor influencia en el proceso corrosivo se ha utilizado el modelo de la Ley Potencial que, de forma genérica, reproduce el proceso electroquímico que se desarrolla en la corrosión atmosférica.

Palabras clave: corrosión atmosférica, cobre, polarización, modelación matemática.

\section{Referencias}

1. L.L. Sheik, Corrosiön, John Wiley \& Sons, Inc., New York, NY, USA, 1963, Vol. 1, 4.27-4.52.

2. E. Mattsson, Br. Corros. J. 15 (N¹) (1980) 6-13.

3. J.E. González González, J.J. Santana Rguez., F.J. Santana Hdez., “Mapa de Corrosión de Canarias - Provincia de Las Palmas, tomo VII. Corrosión Atmosférica del Cobre”, 1999.

4. J.E. González González, J.J. Santana Rguez., F.J. Santana Hdez., “Mapa de Corrosión de Canarias - Provincia de Las Palmas, tomo II. Estudio de los Parámetros Medioambientales", 1999.

5. J.A. Gonzalez Fernández, "Control de la Corrosión. Estudio y Medidas por Técnicas Electroquímicas", Consejo Superior de Investigaciones Cientificas, Centro de Investigaciones Metalúrgicas, Madrid,1989.

6. ASTM G1-90 Norm. "Standard practice for preparing, clearing and evaluating corrosion test specimens".

7. ISO 9223 Norm. "Corrosion of metals and alloys - Corrosivity of atmospheres. Classification".

8. V. Díaz, V. Martínez-Luaces y G. Guineo-Cobs, Rev. Metal. Madrid 39 (2003) 243-251.

9. J.A. González, J.M. Bastidas y S. Feliu, "Corrosión atmosférica del $\mathrm{Al}, \mathrm{Cu}$, Fe y Zn: (III Parte). Posibilidades de las técnicas electroquímicas para estimar la velocidad de corrosión instantánea en atmósferas controladas", Centro Nacional de Investigaciones Metalúrgicas (CENIM), Madrid, 13, (1982).

10. M. Morcillo, S. Feliu, "Mapas de España de Corrosividad Atmosférica", CYTED, Madrid, (1993).

11. "Atmospheric Corrosion", Ed. W.YH. Aila. Reynolds Metals Company, Richmond, Virginia, (1982).

12. I.T.E. Fonseca, R. Picciochi, M.H. Mendonça and A.C. Ramos, Corrosion Science 46 ( N 3) (2004) 547-561.

13. X.M. Zhu and Y.S. Zhang, Corrosion Science 54 (1998).

14. T. Notoya, V. Otieno-Alego and D.P. Schweinsberg, Corrosion Science 37 (1994). 